\title{
FIXED POINT THEOREMS ON INFINITE DIMENSIONAL MANIFOLDS
}

\author{
BY \\ FELIX E. BROWDER(1)
}

Introduction. Let $X$ be a differentiable manifold of finite or infinite dimension, $f$ a continuous mapping of $X$ into $X . f$ is said to be a compact mapping if $f(X)$ is relatively compact in $X$ while $f$ is said to be locally compact if each point $x$ of $X$ has a neighborhood $N_{x}$ such that $f\left(N_{x}\right)$ is relatively compact in $X$.

We are concerned in the present paper with sufficient conditions for the existence of fixed points of compact and locally compact mappings of manifolds, especially those of infinite dimension. The questions which we wish to answer are the following:

(1) If $f$ is a compact self-mapping of an acyclic manifold $X$, does $f$ have a fixed point in $X$ ?

(2) If $f$ is a compact self-mapping of a manifold $X$, does the Lefschetz fixed point theorem hold for $f$ ?

(3) Let $f$ be a locally compact mapping of a manifold $X$ such that for some positive integer $m, f^{m}(X)$ is relatively compact in $X$. Then can we assert that $f$ has a fixed point?

For Banach spaces $X$, the affirmative answer to question (1) is the well-known Schauder fixed point theorem [21]. An affirmative result for question (3) for Banach spaces was obtained by the writer in [3]. For Banach spaces, question (2) is obliquely related to the Leray-Schauder theory of topological degree [20] for completely continuous displacements.

The principal results of the present paper are affirmative answers to questions (1), (2), and (3) for a broad class of manifolds which includes all manifolds which can be imbedded as neighborhood retracts in Banach spaces. More specifically we have the following:

THEOREM (THEOREM 3 OF §1). Let $f$ be a compact mapping of the metric space $X$ where $X$ can be imbedded as a neighborhood retract of a Banach space $E$. Then the Lefschetz number

$$
\Lambda(f)=\sum_{s=0}^{\infty}(-1)^{s} \operatorname{tr}\left(f_{* s}\right)
$$

is well-defined (where $f_{* s}$ is the homomorphism induced by $f$ on $H_{s}(X)$, the $s$-dimensional singular homology group of $X$ with rational coefficients). If $\Lambda(f) \neq 0, f$ has a fixed point in $X$. If $X$ is acyclic, $\Lambda(f)=1$.

Received by the editors September 21, 1964.

(1) The preparation of this paper was partially supported by NSF Grant GP-3552. 
Some examples of acyclic manifolds $X$ satisfying the hypotheses of the above theorem are the following: Let $E$ be an infinite dimensional Banach space, $S(E)$ the unit sphere $\{u \mid u \in E,\|u\|=1\}$ in $E, D(E)$ the open (or closed) unit ball in $E$. Then

$$
\begin{aligned}
& X_{1}=E-\bigcup_{\gamma} Y_{\gamma}, \\
& X_{2}=S(E)-\bigcup_{\gamma} Y_{\gamma}, \\
& X_{3}=D(E)-\bigcup_{\gamma} Y_{\gamma}, \\
& X_{4}=U(H)-\bigcup_{\gamma} Y_{\gamma}
\end{aligned}
$$

are examples, where $H$ is an infinite dimensional Hilbert space, $U(H)$ the unitary group on $H$ in the norm topology, and in each space, $\left\{Y_{\gamma}\right\}$ is a locally finite family of open subsets homeomorphic by $h_{\gamma}$ to open balls in infinite dimensional Banach spaces $E_{\gamma}$ such that $h_{\gamma}$ can be extended to a homeomorphism of the boundaries.

As far as question (3) is concerned we have the following result:

THEOREM (THEOREM 11 OF §2). Let $X$ be a space which can be imbedded as a retract of a space $Y$ such that for each compact subset $K$ of $Y$ there exist a compact acyclic set $A_{1}$ and a compact absolute neighborhood retract $A_{2}$ both containing $K$. (In particular, Y may be any Banach space.) Let $f$ be a locally compact selfmapping of $X$ with $f^{m}(X)$ relatively compact in $X$ for some positive integer $m$.

Then $f$ has a fixed point in $X$.

$X_{1}, X_{2}$, and $X_{3}$ above are examples of spaces satisfying the hypotheses of this last theorem.

$\S 1$ is devoted to the study of compact mappings. We give a general theory of the Lefschetz number for compact mappings of a topological space $X$ which can be imbedded as a retract of a space $X_{1}$ such that every compact subset $K$ of $X$ is contained in a Lefschetz space $Y$ contained in $X_{1}$. ( $Y$ is a Lefschetz space if the Lefschetz fixed point theorem holds for all continuous self-mappings of $Y$.) In Theorem 1, we show that the Lefschetz fixed point theorem holds for all compact mappings of such spaces. In a Corollary, we observe that if such a space is acyclic, every compact self-mapping $f$ of $X$ has a fixed point. The other results of $\S 1$, follow by specialization from Theorem 1 . In Theorems 8 and 9, we show that every closed $C^{3}$ submanifold of a Banach space $E$ admitting $C^{3}$ partitions of unity falls in this class of spaces $\left({ }^{2}\right)$.

(2) Added in proof. The writer has been informed in a recent letter from J. Ellis (commenting upon a preprint of the present paper) that the latter had earlier obtained independently a proof of a result stronger than Theorems 8 and 9 , namely for $C^{0}$ manifolds modeled on $F$-spaces. This result follows directly from Theorem 3 if one uses the known result (which may be derived easily from results of Hanner) that such manifolds may be imbedded as neighborhood retracts of Banach spaces. 
$\S 2$ is devoted to the study of locally compact mappings such that $f^{m}(X)$ is relatively compact for some $m>1$. Here the basic general result is Theorem 11 stated above.

The extensive use of retraction properties in the present paper is related in its general concept to its slightly different use in the study of the local fixed point index by Browder [2], Leray [18], [19], and Deleanu [8]. The proofs of the results of $\$ 2$ are an adaptation and extension of the techniques applied in Banach spaces by the writer in Browder [2], [6], [7]. Other results on the local fixed point index are developed in Leray [17] and Browder [4], [5]. We apply general properties of compact metric absolute neighborhood retracts as given in Kuratowski [13] and Lefschetz [16] and, in particular, our discussion is based on the classical forms of the Lefschetz fixed point theorem for such spaces as established in Lefschetz [15], [16]. In applying our results to particular infinitedimensional manifolds, we make essential use of theorems of Dugundji [9] and of Kuiper [12].

A recent survey of results in general fixed point theory is given by Van der Walt [22].

The writer is indebted to Stephen Smale for stimulating the writer's interest in fixed point theorems for infinite dimensional manifolds and for making him acquainted with some of the results of [1] and [14] applied at the conclusion of $\S 1$.

1. Let $X$ be a topological space. For each integer $s \geqq 0$, we denote by $H_{s}(X)$ the $s$-dimensional singular homology group of $X$ with coefficients in the field $F$ of rational numbers. If $f$ is a continuous mapping of $X$ into $Y$, we denote by $f_{* s}$ the induced homomorphism of $H_{s}(X)$ into $H_{s}(Y) . H_{s}(X)$ and $H_{s}(Y)$ are vector spaces over $F$ and $f_{* s}$ is a linear mapping of $H_{s}(X)$ into $H_{s}(Y)$.

DEFINITION 1. If $V$ is a vector space over $F$ (of possibly infinite dimension), $h$ a linear mapping of $V$ into $V$, then $h$ is said to have a trace if $h(V)$ is of finite dimension. The trace of $h, \operatorname{tr}_{V}(h)$, is defined to be equal to the trace of $h$ restricted to any subspace $V_{1}$ of finite dimension in $V$ which contains $h(V)$.

To deal with the properties of the trace, we need the following well-known elementary fact:

LEMMA 1. Let $V_{1}$ and $V_{2}$ be vector spaces of finite dimension, $h_{1}$ a linear map of $V_{1}$ into $V_{2}, h_{2}$ a linear map of $V_{2}$ into $V_{1}$.

Then

$$
\operatorname{tr}_{V_{1}}\left(h_{2} \circ h_{1}\right)=\operatorname{tr}_{V_{2}}\left(h_{1} \circ h_{2}\right)
$$

DEFINITION 2. Let $f$ be a continuous self-mapping of $X$. Then the Lefschetz number $\Lambda(f)$ of $f$ is said to exist if $f_{* s}=0$ except for a finite number of $s \geqq 0$, while $f_{* s}$ has a trace in the sense of Definition 1 for each $s \geqq 0$.

The Lefschetz number $\Lambda(f)$ is then defined by 


$$
\Lambda(f)=\sum_{s=0}^{\infty}(-1)^{s} \operatorname{tr}\left(f_{* s}\right)
$$

DEFINITION 3. $Y$ is said to be a Lefschetz space if for each continuous selfmapping $f$ of $Y, \Lambda(f)$ is defined and if $\Lambda(f) \neq 0$, then $f$ has a fixed point.

THEOREM 1. Let $f$ be a continuous mapping of the topological space $X$ into $X$. Suppose that there exists a topological space $X_{1}$, a subset $Y$ of $X_{1}$, and an imbedding $j$ of $X$ in $X_{1}$ such that all the following conditions hold:

(a) $j(X)$ is a retract of $X_{1}$,

(b) $Y$ is a Lefschetz space,

(c) $j(f(X)) \subset Y$.

Then $\Lambda(f)$ exists and if $\Lambda(f) \neq 0, f$ has a fixed point in $X$.

Corollary to THeOrem 1. Suppose that in addition to the hypotheses of Theorem 1, we assume also that $X$ is pathwise connected and one of the following conditions hold:

(i) every singular s-cycle in $f(X)$ for $s \geqq 1$ bounds in $X$,

(ii) $f(X)$ is contractible to a point in $X$.

Then $\Lambda(f)=1$ and $f$ has a fixed point.

Before proceeding to the proof of Theorem 1, we remark the following useful fact:

LEMMA 2. If $Y$ is a Lefschetz space, then $H_{s}(Y)$ is of finite dimension for all $s \geqq 0$ and $H_{s}(Y) \neq 0$ only for a finite number of values of $s \geqq 0$.

Proof of Lemma 2. If $v_{Y}$ is the identity map of $Y$, then $\Lambda\left(l_{Y}\right)$ exists. Q.E.D.

Proof of Theorem 1. By identifying $X$ with its image under $j$ in $X_{1}$, we may assume without loss of generality that $X$ is a subset of $X_{1}, j$ is the injection map of $X$ into $X_{1}$, and $f$ maps $X$ into a subset of $X \cap Y$.

Let $k$ be the injection map of $Y$ into $X_{1}, r$ the retraction map of $X_{1}$ on $X$. By considering the image of $x$ in $X$ under $f$ as an element of $Y$ we obtain from $f$ a map $f_{1}$ of $X$ into $Y$. Set

$$
\begin{aligned}
g & =j \circ f \circ r: X_{1} \rightarrow X_{1}, \\
g_{1} & =f_{1} \circ r: X_{1} \rightarrow Y, \\
g_{2} & =g_{1} \circ K: Y \rightarrow Y .
\end{aligned}
$$

Then

$$
g_{* s}=j_{* s} \circ(f \circ r)_{* s}
$$

and since $Y$ is a Lefschetz space, the image of $j_{* s}$ is of finite dimension and $\neq 0$ only for a finite number of values of $s, s \geqq 0$. Hence the same is true for $g_{* s}$, i.e., $\Lambda(g)$ is well-defined. 
Since $f=r \circ g \circ j$, we have for all $s \geqq 0$,

$$
f_{* s}=r_{* s} \circ g_{* s} \circ j_{* s} \text {. }
$$

Since $g_{* s}$ has a finite dimensional image for each $s \geqq 0$, and $g_{* s}=0$ except for a finite number of values of $s$, the same is true of $f_{* s}$. Hence $\Lambda(f)$ exists.

Since $g=j \circ f \circ r$, we have

$$
g_{* s}=j_{* s} \circ f_{* s} \circ r_{* s} .
$$

From the fact that $r \circ j=\imath_{X}$, we have

$$
r_{* s} \circ j_{* s}=1_{* s}
$$

so that $j_{* s}$ is injective and $H_{s}\left(X_{1}\right)$ splits into the sum

$$
H_{s}\left(X_{1}\right)=j_{* s}\left(H_{s}(X)\right)+\operatorname{Ker}\left(r_{* s}\right) \text {. }
$$

On $j_{* s}\left(H_{s}(X)\right), g_{* s}$ may be written as

$$
g_{* s}=j_{* s} f_{* s}\left(j_{* s}\right)^{-1} \text {. }
$$

Hence $\operatorname{tr}\left(g_{* s}\right)=\operatorname{tr}\left(f_{* s}\right)$ for each $s \geqq 0$, so that $\Lambda(g)=\Lambda(f)$.

Suppose now that $\Lambda(f) \neq 0$. Then $\Lambda(g) \neq 0$. On the other hand,

$$
g=k \circ g_{1}
$$

while

$$
g_{2}=g_{1} \circ k \text {. }
$$

Hence

$$
g_{* s}=k_{* s} \circ\left(g_{1}\right)_{* s}
$$

and

$$
\left(g_{2}\right)_{* s}=\left(g_{1}\right)_{* s} \circ k_{* s} \text {. }
$$

By the definition of the trace (Definition 1),

$$
\operatorname{tr}\left(g_{* s}\right)=\operatorname{tr}_{V_{s}}\left(g_{* s}\right)
$$

where $V_{s}=k_{* s}\left(H_{s}(Y)\right) . V_{s}$ is of finite dimension since $Y$ is a Lefschetz space.

We have

and

$$
\begin{aligned}
& \left(g_{1}\right)_{* s}: V_{s} \rightarrow H_{s}(Y), \\
& h_{* s}: H_{s}(Y) \rightarrow V_{s}
\end{aligned}
$$

$$
\begin{aligned}
\left(g_{* s} \mid V_{s}\right) & =k_{s} \circ\left(g_{1}\right)_{* s} \\
\left(g_{2}\right)_{*} & =\left(g_{1}\right)_{* s} \circ k_{s} .
\end{aligned}
$$


Applying Lemma 1 with $V_{1}=V_{s}, V_{2}=H_{s}(Y)$, we find that

$$
\operatorname{tr}\left(\left(g_{2}\right)_{* s}\right)=\operatorname{tr}\left(g_{* s} \mid V_{s}\right)=\operatorname{tr}\left(g_{* s}\right)
$$

so that

$$
\Lambda\left(g_{2}\right)=\Lambda(g)
$$

If $\Lambda(g) \neq 0, \Lambda\left(g_{2}\right) \neq 0$. Since $g_{2}$ is a continuous self-mapping of the Lefschetz space $Y, g_{2}$ has a fixed point $y_{0}$ in $Y$. Hence $y_{0}=f\left(r\left(y_{0}\right)\right)$ so that

$$
y_{0}=f\left(y_{0}\right) \text {. Q.E.D. }
$$

Proof of the Corollary to Theorem 1. The mapping $f$ may be written in the form

$$
f=j_{1} \circ f_{1},
$$

where $f_{1}$ is the obvious map of $X$ into $f(X)$ induced by $f, j_{1}$ the injection map of $f(X)$ into $X$.

If (i) holds, $j_{* s}=0$ for every $s \geqq 1$. Hence $f_{* s}=0$ for every $s \geqq 1$. Thus

$$
\Lambda(f)=\operatorname{tr}\left(f_{* 0}\right)=1,
$$

and the existence of a fixed point for $f$ follows from Theorem 1. Obviously condition (ii) implies condition (i). Q.E.D.

LEMMA 3. The following types of topological spaces are all Lefschet $z$ spaces:

(a) compact metric absolute neighborhood retracts;

(b) convexoid spaces (i.e., compact spaces having arbitrarily fine finite closed coverings each of whose elements is acyclic and such that any nonempty intersection of elements of a given covering is also an element of that covering);

(c) a finite union of compact convex subsets of a Banach space;

(d) a finite union of compact convex subsets of a locally convex linear space $E$;

(e) retracts of Lefschetz spaces.

Proof of Lemma 3. Part (a) was proved by Lefschetz [16]. Part (b) is included in the results of Leray [17].

For the proof in case (c) $\left({ }^{3}\right)$ it suffices, by the result already obtained for case (a), to show that each finite union of compact convex subsets of a Banach space $E$ is a compact metric absolute neighborhood retract (ANR). We note first that by a result of Klee [11], every compact convex subset of a Banach space is homeomorphic to the Hilbert parallelotope and hence is an absolute retract. By a theorem of Aronszajn and Borsuk (cf. [13, p. 260], if $A_{1}$ and $A_{2}$ are two compact ANR's contained in a space $E$ and if $A_{1} \cap A_{2}$ is an ANR, then so is $A_{1} \cup A_{1}$. If $\left\{K_{1}, \cdots, K_{m}\right\}$ is our family of compact convex subsets of $E$, we may assume without loss of generality that each nonempty intersection of a subfamily of

(3) Strictly speaking, the result of (d) includes (c) but we give a simpler and more easily verifiable proof for this case which is the essential one in the applications below. 
the $K_{j}$ 's is also a member of the family and that if $K_{j} \subset K_{k}$, then $j \leqq k$. We then prove by induction on $r(r \leqq m)$ that each union of the form

$$
\bigcup_{k} K_{j(k)} ; \quad j(k) \leqq r
$$

is an ANR. For $r=1$, the result follows from the fact that $K_{1}$ is an absolute retract. Suppose it is true for $(r-1)$. To show that it is true for $r$, we must show that

$$
\left(\bigcup_{j(k)<r-1} K_{j(k)}\right) \cup K_{r}
$$

is an ANR. The union in the round bracket is an ANR by the inductive assumption. We have

$$
K_{r} \cap\left(\bigcup_{j(k) \leqq r-1} K_{j(k)}\right)=\bigcup_{j^{\prime}(k) \leqq-1} K_{j(k)},
$$

which is also an ANR by the inductive assumption. Hence by the theorem of Aronszajn and Borsuk, $\bigcup_{j(k) \leqq} K_{j(k)}$ is also an ANR, the inductive step is proved, and each finite union of compact convex subsets of a Banach space is an ANR.

For the proof in case (d), we observe that each compact convex subset $K$ in a locally convex linear topological space is contractible. Moreover, given an open covering $\alpha$ of $K$, we may find a finite covering $\beta$ of $K$ by closed convex sets which refines $\alpha$. If $\beta=\left\{N_{1}, \cdots, N_{m}\right\}$ then we may replace $N_{j}$ by $K_{j}=N_{j} \cap K$ to obtain a covering by compact convex sets, each of which is contractible and all of whose intersections are contractible. Since contractible sets are acyclic, $K$ is a convexoid space. Moreover, any finite union of $K$ 's may be decomposed as above into a finite union of small compact convex sets. Hence each finite union of compact convex subsets of a locally convex linear space is convexoid. Since convexoid spaces are Lefschetz spaces by part (b), the conclusion of (d) follows.

To prove (e), let $X$ be a Lefschetz space, $Y$ a retract of $X, r$ the retraction map of $X$ on $Y, j$ the injection map of $Y$ into $X$. Since $r \circ j=t_{Y}$, we have $r_{* s} \circ j_{* s}=i_{* s}$ and since the image of $j_{* s}$ is finite dimensional and nontrivial only for finitely many $s \geqq 0$, it follows that $H_{s}(Y)$ is of finite dimension and $H_{s}(Y) \neq 0$ only for finitely many values of $s$. Thus for a self-mapping $f$ of $Y$, $\Lambda(f)$ is always defined.

Let $g=j \circ f \circ r: X \rightarrow X$. For each $s \geqq 0$,

$$
g_{* s}=j_{* s} \circ f_{* s} \circ r_{* s} .
$$

By Lemma 1,

$$
\begin{aligned}
\operatorname{tr}\left(g_{* s}\right) & =\operatorname{tr}\left(j_{* s} \circ\left(f_{* s} \circ r_{* s}\right)\right) \\
& =\operatorname{tr}\left(f_{*} \circ r_{*} \circ j_{*}\right)=\operatorname{tr}\left(f_{*}\right)
\end{aligned}
$$


Hence

$$
\Lambda(f)=\Lambda(g) .
$$

If $\Lambda(f) \neq 0$, we have $\Lambda(g) \neq 0$ so that $g$ has a fixed point $x_{0}$ since $X$ is a Lefschetz space. However

$$
x_{0}=f\left(r\left(x_{0}\right)\right)
$$

so that $x_{0} \in Y, r\left(x_{0}\right)=x_{0}$, and $x_{0}$ is a fixed point of $f$. Q.E.D.

Definition 3. A continuous map $f$ of $X$ into $X$ is said to be compact if $f(X)$ is contained in a compact subset of $X$.

Definition 4. A compact mapping $f$ of $X$ into $X$ is said to be smooth if there exists an imbedding $j$ of $X$ in a space $X_{1}$ such that $j(X)$ is a retract of $X_{1}$ and there exists a Lefschetz space $Y$ contained in $X_{1}$ such that

$$
j(f(X)) \subset Y .
$$

THEOREM 2. Let $f$ be a compact smooth mapping of $X$ into $X$. Then $\Lambda(f)$ exists and if $\Lambda(f) \neq 0, f$ has a fixed point in $X$.

If in addition, every singular cycle in $f(X)$ bounds in $X$ and $X$ is pathwise connected, then $\wedge(f)=1$ and $f$ has a fixed point in $X$.

Proof of Theorem 2. This is just a restatement in the new terminology of Theorem 1 and its Corollary.

THEOREM 3. Let $f$ be a compact mapping of $X$ into $X$ and suppose that $X$ can be imbedded in a Banach space $E$ so that $X$ is the retract of an open neighborhood $U$ of $X$ in $E$. Then $f$ is smooth, $\Lambda(f)$ is well-defined, and if $\Lambda(f) \neq 0$, $f$ has a fixed point in $X$. If $X$ is acyclic $\Lambda(f)=1$.

Proof of Theorem 3. Let $K$ be a compact subset of $X$ containing $f(x)$. It suffices by Theorem 2 to construct a Lefschetz space $Y$ contained in $U$ which contains $K$. We construct $Y$ as the union of a finite number of compact convex subsets of $E$, which is a Lefschetz space by Lemma 3(c). Each point $x$ of $K$ has a closed convex neighborhood $N_{x}$ contained in $U$. A finite family of such neighborhoods $\left\{N_{1}, N_{2}, \cdots, N_{m}\right\}$ covers $K$. Let $K_{j}$ be the convex closure of the compact set $K \cap N_{j} . K_{j}$ is compact and convex, while $K_{i} \subset N_{j} \subset U$. Hence

$$
Y=\bigcup_{j=1}^{m} K_{j} .
$$

is our desired Lefschetz space. Q.E.D.

A similar argument using Lemma 3(d) instead of Lemma 3(c) gives the following result:

THEOREM 4. Let $f$ be a compact continuous self-mapping of the space $X$ and suppose that $X$ may be imbedded as a neighborhood retract in the locally 
convex linear topological space $E$, where $E$ is quasi-complete (i.e., bounded closed sets of $E$ are complete). Then $f$ is smooth, $\Lambda(f)$ exists, and if $\Lambda(f) \neq 0$, $f$ has a fixed point in $X$.

THEOREM 5. Let $E$ be an infinite dimensional Banach space, $S(E)$ its unit sphere

$$
S(E)=\{u \mid u \in E,\|u\|=1\} .
$$

Let $f$ be a compact mapping of $S(E)$ into itself. Then $f$ has a fixed point.

Proof of Theorem 5. By a theorem of Dugundji [9], there exists a retraction of the closed disk $D(E)=\{u \mid u \in E,\|u\| \leqq 1\}$ onto $S(E)$. Since $D(E)$ is a neighborhood retract in $E$ and is contractible, it follows by Theorem 3 that $f$ is smooth, $\Lambda(f)$ exists, and $S(E)$ is acyclic.

Hence $\Lambda(f)=1$ and $f$ has a fixed point. Q.E.D.

THEOREM 6. Let $X$ be any one of the following:

(a) an infinite dimensional Banach space $E$.

(b) $S(E)$,

(c) $D(E)$,

(d) an acyclic neighborhood retract in a Banach space $E$.

Suppose that $\left\{Y_{\gamma}\right\}$ is a locally finite family of open subsets of $X$ which are pairwise disjoint and such that for each $\gamma, \bar{Y}_{\gamma}$ (the closure of $Y$ in $X$ ) is homeomorphic to $D\left(E_{\gamma}\right)$ for some infinite dimensional Banach space $E_{\gamma}$ under a homeomorphism $h$ which carries $S\left(E_{\gamma}\right)$ onto the boundary $Y$ of $Y_{\gamma}$ in $X$.

Let $X_{2}=X-\bigcup_{\gamma} Y_{\gamma}$ and let $f$ be a compact map of $X_{2}$ into $X_{2}$. Then $f$ has a fixed point in $X_{2}$.

Proof of Theorem 6. By Theorem 3, it suffices to show that $X_{2}$ is a retract of $X$. We define the retraction $r$ by noting that by the theorem of Dugundji [9] applied above, $\dot{Y}_{\gamma}$ is a retract of $\bar{Y}_{\gamma}$. Q.E.D.

THEOREM 7. Let $H$ be an infinitely dimensional Hilbert space, $U(H)$ the group of unitary operators on $H$ in the norm topology. Let $f$ be a compact continuous map of $U(H)$ into itself. Then $f$ has a fixed point.

Proof of Theorem 7. Let $L(H)$ be the space of bounded linear operators on $H$ with the norm topology, $G L(H)$ the open subset of $L(H)$ consisting of invertible operators, $U(H)$ the subset of unitary operators. To prove Theorem 6 , it suffices by Theorem 2 and 3 to prove that $U(H)$ is a retract of $G L(H)$ and that for $s \geqq 1$, each singular cycle in $U(H)$ bounds. The second fact follows from a recent result of $\mathrm{N}$. Kuiper [12] that $\pi_{j}(U(H))=0$ for all $j \geqq 1$ together with the Hurewicz theorem. For the first, we remark that every $A$ in $G L(H)$ may be written uniquely in the form 


$$
A=U|A|
$$

where $|A|=\left(A^{*} A\right)^{1 / 2}$. If the map $A \rightarrow U$ is continuous in norm, it provides the necessary retraction.

To prove that the mapping $A \rightarrow U$ is norm continuous, it suffices since $U=A|A|^{-1}$ to show that the map $A \rightarrow|A|$ is continuous in norm. Since $|A|^{2}=A^{*} A$ where the latter is norm-continuous in $A$, it suffices to show that the map $|A|^{2} \rightarrow|A|$ is norm-continuous or more generally that over the set of bounded self-adjoint operators $B$ with $B \geqq I$, the mapping $B \rightarrow B^{1 / 2}$ is normcontinuous.

Let $C$ be a smooth Jordan curve in the complex plane which contains the segment $\left[1,\left\|B_{0}\right\|\right]$ in the interior and is symmetric under the map $\zeta \rightarrow \bar{\zeta}$. For selfadjoint operators $B$ close to $B_{0}$ in norm, the spectrum of $B$ will lie inside $C$. We form

$$
B_{1}=\frac{1}{2 \pi} \bar{i} \int_{C} \lambda^{1 / 2}(\lambda I-B)^{-1} d \lambda
$$

Then $B_{1}^{*}=B_{1}, B_{1}^{2}=B$ and $B_{1} \geqq 0$. Hence $B_{1}$ is the unique positive square root of $B$. It follows easily from the integral formula that $B_{1}=B^{1 / 2}$ is norm continuous in $B$. Q.E.D.

By an infinite dimensional manifold modeled on the Banach space $E$ is meant (Lang [14]) a Hausdorff space $X$ carrying an atlas of coordinate charts on $B$, i.e., with a given family $\left\{U_{j}, \phi_{j}\right\}$ where $U_{j}$ is an open subset of $X$ and $\phi_{j}$ is a homeomorphism of $U_{j}$ on the open unit ball $B$ in $E(B=\{u \mid u \in E,\|u\|<1\})$. $X$ is said to be a manifold of class $C^{s}, s \geqq 1$, if for each pair of charts $\left(U_{j}, \phi_{j}\right)$ and $\left(U_{k}, \phi_{k}\right)$ such that $U_{j} \cap U_{k} \neq \varnothing$, the mapping $\phi_{k} \phi_{j}^{-1}$ is a diffeomorphism of class $C^{s}$ of $\phi_{j}\left(U_{j} \cap U_{k}\right)$ onto $\phi_{k}\left(U_{j} \cap U_{k}\right)$. If $X$ and $Y$ are manifolds of class $C^{s}$, the continuous mapping $f$ of $X$ into $Y$ is said to be of class $C^{s}$ if for each coordinate chart $\left(U_{j}, \phi_{j}\right)$ on $X$ and $\left(V_{k}, \psi_{k}\right)$ on $Y$ such that $f\left(U_{j}\right) \cap V_{k} \neq \varnothing$, the mapping

$$
\psi_{k} \circ f \circ \phi_{j}^{-1}
$$

is a mapping of class $C^{s}$ from $\phi_{j}\left(U_{j} \cap f^{-1}\left(V_{k}\right)\right)$ into $\psi_{k}\left(V_{k}\right)$.

If $X$ is a manifold of class $C^{s}, s \geqq 1, x$ a point of $X$, we consider curves of class $C$ starting at $x$ (i.e., $C^{s}$-maps $g$ of the unit interval $[0,1]$ into $X$ such that $g(0)=x)$. Two such curves $g$ and $g_{1}$ are said to be equivalent if for a coordinate chart $(U, \phi)$ at $x, \phi \circ g$ and $\phi \circ g_{1}$ have the same tangent vector at 0 . The tangent space $T_{x}$ to $X$ at $x$ consists of the set of equivalence classes of curves in $X$ at $x$ with a linear structure obtained from its identification with the tangent vectors of the curves $\phi \circ g$ at $\phi(x)$ in $E$. To each differentiable map $f$ of $X$ into $Y$, there corresponds a linear map $d f_{x}$ of $T_{x}$, the tangent space to $X$ at $x$, into $T_{f(x)}$, the 
tangent space to $Y$ at $f(x)$, where $d f_{x}$ assigns to the equivalence class of curve $g:(I, 0) \rightarrow(X, x)$ in $X$, the equivalence class of the curve $f \circ g:(I, 0) \rightarrow(Y, f(x))$.

A mapping $h$ of $X$ into $Y$ of class $C^{s}, s \geqq 1$, is said to be an imbedding of $X$ as a submanifold of class $C^{s}$ in $Y$ if $h$ is homeomorphism of $X$ on $h(X)$ and if for each point $x$ of $X, d f_{x}$ is an injective map of $T_{x}$ into $T_{f(x)}$. We say that $X$ is imbedded as a closed submanifold of $Y$ if $h(X)$ is closed in $Y$. An open neighborhood $U$ of $X$ in $Y$ is said to be a tubular neighborhood if there exists a fibre bundle $M$ over $X$ whose fibre is a Banach space and an open neighborhood $V$ of the zero section of $M$ which is homeomorphic to $U$. We may obviously assume that $X$ is a deformation retract of $U$.

THEOREM 8. Let $X$ be a closed $C^{3}$-submanifold of the Banach space $E$, where $E$ admits $C^{2}$ partitions of unity. Then $X$ is a neighborhood retract of $E$ and for every compact mapping $f$ of $X$ into $X, \Lambda(f)$ exists and the Lefschetz fixed point theorem is valid for $f$.

Proof of Theorem 8. The retraction property follows from the theorem on the existence of a tubular neighborhood of $X$ in $E$ under the hypotheses of Theorem 8 [14], [1, p. 73]. The remaining conclusion follows from Theorem 3. Q.E.D.

THEOREM 9. Every paracompact separable $C^{3}$-manifold modeled on a separable Banach space $E_{1}$ with $C^{3}$ partitions of unity can be $C^{3}$-imbedded as a closed submanifold of a Banach space $E$ with $C^{3}$ partitions of unity. For such a manifold, the conclusion of Theorem 8 holds.

Proof of Theorem 9. The proof is essentially identical with the proof of Theorem 7.4 of [1] for the case where $E_{1}$ is a separable Hilbert space.

2. In the present section, we establish the extension of some of the results of $\S 1$ to mappings $f$ which need no longer be compact but such that $f$ is locally compact and $f^{m}$ is compact for some integer $m>1$. The results obtained below generalize and strengthen corresponding results of the writer [3], [6], [7] for self-mappings of Banach spaces.

Our basic tool is the following theorem, which was stated without proof as Theorem 3 of [7].

THEOREM 10. Let $X_{0}$ be a compact absolute neighborhood retract, $X_{1}$ an open subset of $X_{0}, X_{2}$ a compact subset of $X_{1}$. Let $f$ be a continuous mapping of $X_{1}$ into $X_{0}, m$ a positive integer such that $f^{m}$ is defined on $X_{2}$ and

$$
f^{m}\left(\bigcup_{j=0}^{m-1} f^{j}\left(X_{2}\right)\right) \subset X_{3} \subset \operatorname{Int}\left(X_{2}\right)
$$

(where $\operatorname{Int}\left(X_{2}\right)$ is the interior of $X_{2}$ with respect to $\left.X_{0}\right)$ and $X_{3}$ is acyclic.

Then $f$ has a fixed point in $X_{2}$. 
Proof of Theorem 10. Let $\Omega$ be the directed set of finite open coverings $\alpha$ of $X_{0}$. For each $\alpha$ in $\Omega$, let $N_{\alpha}$ denote the nerve of the covering $\alpha$, so that each vertex $v$ of the finite polytope $N_{\alpha}$ corresponds to an element $V_{v}$ of $\alpha$. For each $\alpha$, we choose a canonical mapping $p_{\alpha}$ of $X_{0}$ into $N_{\alpha}$.

For each $\varepsilon>0$, there exists $\delta>0$ such that if $\alpha \in \Omega$ with $\operatorname{mesh}(\alpha)=\sup _{V \in \alpha}$ $\operatorname{diam}(V)<\delta$, then there exists a continuous map $q_{\alpha}$ of $N_{\alpha}$ into $X_{0}$ such that the mapping $q_{\alpha} \circ p_{\alpha}$ moves each point of $X_{0}$ at most $\varepsilon$ and is $\varepsilon$-homotopic to $\mathbf{l}_{X_{0}}$ the identity map of $X_{0}$. For a given $\varepsilon>0$, we choose such a mapping $q_{\alpha}$ for each $\alpha$ with mesh $<\delta$, where $\varepsilon$ will be specified at a later point of the proof.

Let $N_{\alpha}^{\prime}$ be the closed subcomplex of $N_{\alpha}$ of all simplexes of $N_{\alpha}$ all of whose vertices correspond to elements $V$ of $\alpha$ whose stars in $\alpha$ are contained in $X_{1}-M_{\varepsilon}\left(X_{0}-X_{1}\right)$ where $M_{\varepsilon}(S)$ is the $\varepsilon$-neighborhood of $S$ in $X_{0}$. Then $q_{\alpha}$ maps $N_{\alpha}^{\prime}$ into $X_{1}$ and we may form the mapping

$$
f_{\alpha}: N_{\alpha}^{\prime} \rightarrow N_{\alpha}
$$

given by

$$
f_{\alpha}=p_{\alpha} \circ f \circ\left(q_{\alpha} \mid N_{\alpha}^{\prime}\right) \text {. }
$$

Let $N_{\alpha}^{\prime \prime}$ be the closed subcomplex of $N_{\alpha}$ of all simplexes all of whose vertices correspond to elements $V$ of $\alpha$ which intersect $M_{\varepsilon}\left(X_{2}\right)$.

We verify the following facts about $f_{\alpha}, N_{\alpha}^{\prime}$, and $N_{\alpha}^{\prime \prime}$ if $\varepsilon>0$ is sufficiently small and $\alpha \in \Omega$ is sufficiently fine:

(1) $f_{\alpha}^{m}$ is defined on $N_{\alpha}^{\prime \prime}, f_{\alpha}^{m}\left(N_{\alpha}^{\prime \prime}\right) \subset N_{\alpha}^{\prime \prime}$ and

$$
f_{\alpha}^{m}\left(\bigcup_{j=0}^{m-1}\left(f_{\alpha}\right)^{j}\left(N_{\alpha}^{\prime \prime}\right)\right) \subset \operatorname{Int}\left(N_{\alpha}^{\prime \prime}\right)
$$

(where Int $\left(N_{\alpha}^{\prime \prime}\right)$ is the interior of $N_{\alpha}^{\prime \prime}$ with respect to $N_{\alpha}^{\prime \prime}$ );

(2) $N_{\alpha}^{\prime \prime}$ lies in the interior of $N_{\alpha}^{\prime}$;

(3) $f_{\alpha}^{m}$ mapping $\bigcup_{j=0}^{m-1}\left(f_{\alpha}\right)^{j}\left(N_{\alpha}^{\prime \prime}\right)$ into $N_{\alpha}^{\prime \prime}$ is homologically trivial since it is the composition of a homologically trivial map $h_{\alpha}$ into $X_{2}$ with the map $p_{\alpha}$ of $X_{2}$ into $N_{\alpha}^{\prime \prime}$. Here we may take $h_{\alpha}=f \circ q_{\alpha} f_{\alpha}^{m-1} \cong f^{m} \circ q_{\alpha}$.

Using (1), (2), and (3), we have essentially reduced the proof of Theorem 10 to the case when $X_{0}, X_{1}$, and $X_{2}$ are finite polytopes. We now perform a further reduction by replacing $f_{\alpha}$ by another mapping $g_{\alpha}$ of $N_{\alpha}^{\prime}$ into $N_{\alpha}$ where $g_{\alpha}$ is a simplicial mapping of one barycentric subdivision $B_{r}\left(N_{\alpha}^{\prime}\right)$ into another $B_{s}\left(N_{\alpha}\right)$ such that

(1') $g_{\alpha}^{m}$ is defined on $N_{\alpha}^{\prime \prime}, g_{\alpha}^{m}\left(N_{\alpha}^{\prime \prime}\right) \subset N_{\alpha}^{\prime \prime}$;

(2') $g_{\alpha}^{m}\left(\bigcup_{j=0}^{m-1}\left(g_{\alpha}\right)^{j}\left(N_{\alpha}^{\prime \prime}\right)\right) \subset \operatorname{Int}\left(N_{\alpha}^{\prime \prime}\right)$;

(3') $g_{\alpha}^{m}$ mapping $\bigcup_{j=0}^{m-1}\left(g_{\alpha}^{\prime}\right)^{j}\left(N_{\alpha}^{\prime \prime}\right)$ into $N_{\alpha}^{\prime \prime}$ is homologically trivial;

(4) $g_{\alpha}^{j}, 0 \leqq j \leqq m-1$, is a simplicial map from the barycentric subdivision of $N_{\alpha}$ to another. 
We set

$$
A_{\alpha}=\bigcup_{j=0}^{m-1}\left(g_{\alpha}\right)^{j}\left(N_{\alpha}^{\prime \prime}\right) .
$$

By property (4), $A_{\alpha}$ is a finite union of simplexes and hence an absolute neighborhood retract. By property ( $\left.1^{\prime}\right), g_{\alpha}$ maps $A_{\alpha}$ into $A_{\alpha}$. By property (3'), $g_{\alpha}^{m}$ is a homologically trivial map of $A_{\alpha}$ into $A_{\alpha}$.

LEMMA (2.1) (LEMMA 1 of [3]). If $A$ is a compact absolute neighborhood retract, $f$ a continuous self-mapping of $A$ such that $f^{m}$ is homologically trivial, then $f$ has a fixed point on $A$.

Proof of Lemma (2.1). Since $0=\left(f^{m}\right)_{* s}=\left(f_{* s}\right)^{m}$ for $s \geqq 1$, we know that $\operatorname{tr}\left(f_{* s}\right)=0$ for $s \geqq 1$. Since $\left(f^{m}\right)_{* s}$ is the identity on one generator of $H_{0}(A)$, zero on the rest, $\operatorname{tr}\left(f_{0}\right)=1$. Hence $\Lambda(f)=1$ and $f$ has a fixed point by the Lefschetz fixed point theorem.

Proof of Theorem 10 completed. By Lemma (2.1), $g_{\alpha}$ has a fixed point in $A_{\alpha}$ which must lie in $N_{\alpha}^{\prime \prime}$. If $w_{\alpha}$ is such a fixed point, $w$ and $f_{\alpha}\left(w_{\alpha}\right)$ must lie in the same simplex of $N_{\alpha}$. Hence if $x_{\alpha}=g_{\alpha}\left(w_{\alpha}\right)$,

$$
\operatorname{dist}\left(x_{\alpha}, g_{\alpha} f_{\alpha}\left(w_{\alpha}\right)\right)=\left(g_{\alpha} \circ p_{\alpha}\right)\left(f\left(x_{\alpha}\right)\right)
$$

is at most at distance $\varepsilon$ from $f\left(x_{\alpha}\right)$. Hence if $3 \operatorname{mes}(\alpha)<\varepsilon$, we have

$$
\operatorname{dist}\left(x_{\alpha}, f\left(x_{\alpha}\right)\right)<2 \varepsilon .
$$

Since $\varepsilon>0$ can be made arbitrarily small, there must exist a fixed point $x_{0}$ of $f$ on $X_{2}$ (since all the fixed points $x_{\alpha}$ constructed above lie in the $3 \varepsilon$-neighborhood of $X_{2}$ in $X_{0}$ ). Q.E.D.

DEFINITION (2.1). Let $X$ be a topological space, $f$ a continuous self-mapping of $X$. Then $f$ is said to be locally compact if each point $x$ of $X$ has a neighborhood $N_{x}$ in $X$ such that $f\left(N_{x}\right)$ is relatively compact in $X$.

THEOREM 11. Let $X$ be a topological space, $f$ a locally compact self-mapping of $X$. Suppose that $X$ has an imbedding $j$ in a space $Y$ such that $j(X)$ is a retract of $Y$ which for each compact subset $K$ of $Y$ there exist sets $A_{1}$ and $A_{2}$ in $Y$ containing $K$ where $A_{1}$ is compact and acyclic while $A_{2}$ is a compact absolute neighborhood retract. Suppose that for some integer $m \geqq 1, f^{m}(X)$ is relatively compact in $X$.

Then $f$ has a fixed point in $X$.

Proof of Theorem 11. We may assume without loss of generality that $X$ is a subset of $Y$ and $j$ is the injection map of $X$ into $Y$. We may also assume without loss of generality that $X=Y$ since if $r$ is the retraction map of $Y$ onto $X$, then the self-mapping $g=j \circ f \circ r$ of $Y$ satisfies all the hypotheses imposed on $f$ and has the same fixed points as $f$. 
Since $f^{m}(X)$ is relatively compact in $X$, its closure $K_{0}$ in $X$ is compact. So is

$$
K=\bigcup_{j=0}^{m-1} f^{j}\left(K_{0}\right)
$$

and we see that $f(K) \subset K$.

Since we are assuming that $X=Y$, it follows from the hypothesis of Theorem 11 that there exists a compact acyclic set $A_{1}$ in $X$ which contains $K$. Let

$$
A_{1}^{\prime}=\bigcup_{j=0}^{m-1} f^{j}\left(A_{1}\right)
$$

Since $A_{1}^{\prime}$ is compact and $f$ is a locally compact mapping, there exists a neighborhood $U$ of $A_{1}^{\prime}$ in $X$ such that $f(U)$ is contained in a compact subset $K^{\prime}$ of $X$. By the hypothesis of Theorem 11, there exists a compact absolute neighborhood retract $A_{2}$ in $X$ which contains $A_{1}^{\prime} \cup K^{\prime}$.

We now apply Theorem 10 taking $X_{0}=X_{2}, X_{1}=A_{2} \cap f^{-1}\left(A_{2}\right)$ and considering the mapping $f_{1}$ of $X_{1}$ into $X_{0}$ obtained by restricting $f$ to $f^{-1}\left(A_{2}\right) \cap A_{2}$ and taking the restricted mapping as a mapping into $A_{2}$. We set $X_{3}=A_{1}$. Since $f^{m}(X) \subset A_{1}$, it will certainly follow that $f_{1}^{m}$ will map the domain of $f_{1}^{m}$ into $A_{1}$. We note that $A_{1}$ lies in the domain of $f_{1}^{m}$ since $f^{j}$ maps $A_{1}$ into $A_{2}$ for all $j \leqq m$. Furthermore, the domain of $f_{1}$ contains $A_{1}$ in its interior since

$$
A_{1} \subset U \cap A_{2} \subset f^{-1}\left(A_{2}\right) \cap A_{2}
$$

and $U \cap A_{2}$ is open in $A_{2}$. Let $U_{1}$ be the interior of the domain of $f_{1}$. Then for each $j$ with $0 \leqq j \leqq m-1$

$$
f_{1}^{-1}\left(U_{1}\right)
$$

is open in $A_{2}$ and contains $A_{1}$. Hence

$$
A_{1} \subset \bigcap_{j=0}^{m-1} f_{1}^{-j}\left(U_{1}\right)
$$

where $\bigcap_{j=0}^{m-1} f_{1}^{-j}\left(U_{1}\right)$ is an open subset of the domain of $f_{1}^{m}$. Hence $A_{1}$ lies in the interior of the domain of $f_{1}^{m}$.

Let $X_{2}$ be a compact subset of the domain of $f_{1}^{m}$ which contains $A_{1}=X_{3}$ in its interior. Then the family $\left(X_{0}, X_{1}, X_{2}, X_{3}, f_{1}\right)$ satisfies the hypotheses of Theorem 10 so that $f_{1}$ has a fixed point in $X_{2}$. Since $f_{1}$ is a restriction of $f, f$ has a fixed point in $X$. Q.E.D.

We now obtain some specializations of Theorem 11:

THEOREM 12. Let $X$ be one of the following spaces:

(1) $X=E-\bigcup_{\gamma} Y_{\gamma}$, where $E$ is an infinite dimensional Banach space, $\left\{Y_{\gamma}\right\}$ a locally finite family of mutually disjoint open balls of $E$. 
(2) $X=S(E)-\bigcup_{\gamma} Y_{\gamma}$, where $S(E)$ is the unit sphere in an infinite-dimensional Banach space $E,\left\{Y_{\gamma}\right\}$ a locally finite family of mutually disjoint open subsets of $S(E)$ such that for each $\gamma$ there exists a homeomorphism $h_{\gamma}$ of $Y_{\gamma}$ on an open ball $D\left(E_{\gamma}\right)$ in an infinite-dimensional Banach space $E_{\gamma}$ which can be extended to a homeomorphism of $\bar{Y}_{\gamma}$ on the closed ball mapping $\dot{Y}_{\gamma}$ homeomorphically on $S\left(E_{\gamma}\right)$.

(3) $X=D(E)-\bigcup_{\gamma} Y_{\gamma}$, where $D(E)$ is the open (or closed) unit ball in an infinite-dimensional Banach space $E$ and $Y_{\gamma}$ is a mutually disjoint locally finite family of open subsets of $D(E)$ of the same type as in (2).

Let $f$ be a locally compact self-mapping of $X$ such that for some $m \geqq 1, f^{m}(X)$ is relatively compact in $X$.

Then $f$ has a fixed point in $X$.

Proof of Theorem 12. It suffices by Theorem 11 to show that each of the types of spaces $X$ of Theorem 12 can be imbedded as a retract of a convex subset of a Banach space which is either open or closed. We apply the theorem of Dugundji [9] that in an infinite-dimensional Banach space $E, S(E)$ is a retract of the closed unit ball $D(E)$. Hence $E, D(E)$ and $S(E)$ all satisfy the conditions of Theorem 11. Let $X_{0}$ be one of these and let $X=X_{0}-\bigcup_{\gamma} Y_{j}$ where $\left\{Y_{\gamma}\right\}$ is a family satisfying the conditions of (2) of the hypothesis of Theorem 12. By Dugundji's theorem, there exists a retraction of $Y_{\gamma} \cup \dot{Y}_{\gamma}$ on $\dot{Y}_{\gamma}$ and hence a retraction of $X_{0}$ on $X$. Since a retract of a retract is a retract, our proof is complete. Q.E.D.

\section{BIBLIOGRAPHY}

1. R. Abraham, Lectures of Smale on differential topology, Columbia Univ. Notes, New York, 1963.

2. F. E. Browder, The topological fixed point theory and its applications to functional analysis, Ph. D. Dissertation, Princeton University, Princeton, N. J., 1948.

3. - On a generalization of the Schauder fixed point theorem, Duke Math. J. 26 (1959), 291-303.

4. - On the fixed point index for continuous mappings of locally connected spaces, Summa Brasil. Math. 4 (1960), 253-293.

5. - On continuity of fixed points under deformations of continuous mappings, Summa Brasil. Math. 4 (1960), 183-190.

6. - Another generalization of the Schauder fixed point theorem, Duke Math. J. (to appear).

7. —_, A further generalization of the Schauder fixed point theorem, Duke Math. J. (to appear).

8. A. Deleanu, Théorie des points fixes sur les retractes de voisinage des espaces convexoides, Bull. Soc. Math. France 87 (1959), 235-243.

9. J. Dugundji, An extension of Tietze's theorem, Pacific J. Math. 1 (1951), 353-367.

10. D. Göhde, Uber Fixpunktsätze und die Theorie des Abbildungsgrades in Funktionalraumen, Math. Nachr. 20 (1959), 356-371.

11. V. L. Klee, Some topological properties of convex sets, Trans. Amer. Math. Soc. 78 (1955), 30-45.

12. N. H. Kuiper, The general linear group of Hilbert space is $k$-connected (to appear). 
13. K. Kuratowski, Topologie, Vol. 2, Warsaw, 1961.

14. S. Lang, Introduction to differentiable manifolds, Interscience, New York, 1962.

15. S. Lefschetz, Algebraic topology, Amer. Math. Soc. Colloq. Publ. Vol. 27, Amer. Math. Soc., Providence, R. I., 1945.

16. —- Topics in topology, Annals of Mathematics Studies No. 10, Princeton Univ. Press, Princeton, N. J., 1942.

17. J. Leray, Sur les equations et les transformations, J. Math. Pure Appl. 24 (1945), 201-248.

18. —_L La théorie des points fixes et ses applications en analyse, Proc. Internat. Congress Math., 1950, Cambridge, Mass., Vol. 2, pp. 202-208.

19. - Théorie des points fixes, indice total, et nombre de Lefschetz, Bull. Soc. Math. France, 87 (1959), 221-233.

20. J. Leray et J. Schauder, Topologie et équations fonctionelles, Ann. Sci. École Norm. Sup. 51 (1934), 45-78.

21. J. Schauder, Der Fixpunksatz in Funktionalraum, Studia Math. 2 (1930), 171-180.

22. T. H. Van der Walt, Fixed and almost fixed points, Mathematical CenterTracts No. 1, Amsterdam, 1963.

\section{UNIVERSITY OF CHICAGo, \\ Chicago, Illinois}

